\title{
MIR34B Pre-miRNA
}

National Cancer Institute

\section{Source}

National Cancer Institute. MIR34B Pre-miRNA. NCI Thesaurus. Code C82730.

MIR34B is an oligoribonucleotide that is encoded by the human MIR34B gene and has a

role in the regulation of gene expression. 